\title{
Dental Liner
}

National Cancer Institute

\section{Source}

National Cancer Institute. Dental Liner. NCI Thesaurus. Code C42795.

Material applied to the dental tissue or to the tissue side of a denture to provide biological, chemical and physical protection. 